\title{
Pour une contribution du management stratégique à l'écologie industrielle
}

For a contribution of strategic management to industrial ecology

\section{Anne-Ryslène Zaoual}

\section{OpenEdition}

1 Journals

\section{Édition électronique}

URL : https://journals.openedition.org/developpementdurable/10220

DOI : 10.4000/developpementdurable. 10220

ISSN : 1772-9971

Éditeur

Association DD\&T

\section{Référence électronique}

Anne-Ryslène Zaoual, « Pour une contribution du management stratégique à l'écologie industrielle », Développement durable et territoires [En ligne], Vol. 5, nº 1 | Février 2014, mis en ligne le 04 février 2014, consulté le 21 septembre 2021. URL : http://journals.openedition.org/developpementdurable/10220 ; DOI : https://doi.org/10.4000/developpementdurable.10220

\section{Ce document a été généré automatiquement le 21 septembre 2021.}

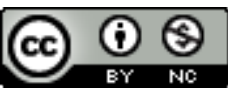

Développement Durable et Territoires est mis à disposition selon les termes de la licence Creative Commons Attribution - Pas d'Utilisation Commerciale 4.0 International. 


\title{
Pour une contribution du management stratégique à l'écologie industrielle
}

\author{
For a contribution of strategic management to industrial ecology
}

\author{
Anne-Rysléne Zaoual
}

1 La raréfaction des ressources naturelles (en amont) et la pollution croissante (en aval) des processus de production révèlent les limites du système industriel linéaire, considéré comme "primitif» et «immature» (Ayres, 1989; Tranchant et al., 2004). Ces défis environnementaux mais aussi le développement des mouvements écologistes et l'omniprésence des médias obligent désormais les entreprises à intégrer l'environnement dans leurs orientations stratégiques. Pour répondre à ces pressions tout en restant compétitives, les organisations cherchent à développer des stratégies alliant performance et respect de l'environnement.

2 Officiellement née dans les années $1990^{1}$, l'écologie industrielle suggère l'« écorestructuration" (Tranchant et al., 2004) du système industriel sur le modèle des écosystèmes naturels dont le fonctionnement est cyclique (Ayres, 1989; Frosch et Gallopoulos, 1989 ; Allenby, 1992 ; Brown, 2003). En considérant notamment les déchets ${ }^{2}$ générés par les uns comme des matières premières potentielles pour les autres ${ }^{3}$, elle vise, in fine, la création d'écosystèmes industriels. Ce concept s'est progressivement imposé au sein de la communauté académique mais également auprès des entreprises et des acteurs institutionnels.

3 La majorité des travaux sur l'écologie industrielle se concentre sur les flux physiques de matière et d'énergie (Korhonen, 2004). L'intérêt d'y intégrer les sciences humaines a néanmoins été souligné (Boons et Baas, 2006; Baas, 2007). Si certains enjeux de l'écologie industrielle, notamment ses avantages et ses inconvénients pour l'entreprise sont présentés dans la littérature, d'autres problématiques organisationnelles gagneraient à 
être étudiées en profondeur. Cette communication vise à souligner les contributions potentielles du management stratégique au champ de l'écologie industrielle.

Structurée en trois étapes, cette contribution entend susciter et inspirer de nouveaux questionnements et de nouvelles recherches. Elle débute par une présentation du management stratégique et décrit son intérêt pour l'écologie industrielle (§.1). Elle se poursuit par un recensement de ses principaux avantages et inconvénients pour l'entreprise (\$.2). La mise en relief de ces problématiques opérationnelles conduit enfin à la proposition d'un agenda de recherche sur l'écologie industrielle en mobilisant le champ du management stratégique (\$.3).

\section{Pour une approche stratégique de l'écologie industrielle}

5 Ouvrages, conférences, expériences,... Depuis une vingtaine d'années, le concept d'écologie industrielle connaît un certain essor. La configuration d'un système d'alertes sur Google pour le terme "écologie industrielle » permet d'apprécier son succès, notamment sur Internet. La réception quotidienne de notifications confirme en effet sa mobilisation croissante et sa large diffusion ces dernières années.

6 Les appels à communication et à projets se multiplient également. De nombreuses disciplines sont désormais sollicitées pour apporter un éclairage aux pratiques d'écologie industrielle: géographie, économie, sociologie, anthropologie, politique, écologie, biologie, chimie, ingénierie, etc. Si la nécessité de mobiliser les sciences humaines est reconnue (Boons et Baas, 2006 ${ }^{4}$; Baas, 2007), le chercheur en stratégie reste souvent absent. À cet égard, Brent et al. (2008:2, notre traduction) soulignent que «le champ de l'écologie industrielle a été développé sans perspective stratégique ».

7 Le terme "stratégie », du grec strategos désignant un officier militaire supérieur (général, chef d'armée), correspond à l'art de diriger une armée face à l'ennemi. "Le mot stratégie évoque donc la guerre (contre les concurrents) et le leadership (du dirigeant) qui doit conduire une armée bien organisée (l'entreprise) à la victoire (la performance économique) tout en préservant au maximum la vie des soldats (les salariés)." précisent Ardoin et al. (2009: 5). En quelques décennies, la stratégie est devenue une discipline majeure en sciences de gestion (Desreumaux et al., 2009). Désormais institutionnalisé et reconnu, le champ du management stratégique s'est doté de revues spécialisées, d'associations et de congrès spécifiques (Desreumaux, 2008).

8 De nombreuses définitions ont été proposées. Si aucune n'est acceptée unanimement, les auteurs se rejoignent sur plusieurs points (tableau 1).

Tableau 1. Quelques définitions de la stratégie

\begin{tabular}{|l|l|}
\hline \multicolumn{2}{|l|}{ Définitions de la stratégie } \\
\hline $\begin{array}{l}\text { Von Neumann et } \\
\begin{array}{l}\text { Morgenstern } \\
(\mathbf{1 9 4 7 )}\end{array}\end{array}$ & "La stratégie est l'ensemble des actions décidées par une entreprise en fonction \\
d'une situation particulière. »
\end{tabular}




\begin{tabular}{|c|c|}
\hline Chandler (1962) & $\begin{array}{l}\text { "La stratégie est la détermination des buts à long terme de l'entreprise et le choix } \\
\text { des actions et de l'allocation des ressources nécessaires à leur atteinte." }\end{array}$ \\
\hline $\begin{array}{l}\text { Labourdette } \\
\text { (1989) }\end{array}$ & $\begin{array}{l}\text { "La définition d'actions cohérentes cherchant à atteindre dans le temps des buts } \\
\text { hiérarchisés." }\end{array}$ \\
\hline Bent (1990) & $\begin{array}{l}\text { Le but de la stratégie est "d'associer des ressources dans des schémas d'action } \\
\text { intégrés, en vue d'obtenir des avantages concurrentiels bien marqués et } \\
\text { d'atteindre des buts déterminés." }\end{array}$ \\
\hline Thiétart (1990) & $\begin{array}{l}\text { "La stratégie est l'ensemble des décisions et des actions relatives au choix des } \\
\text { moyens et à l'articulation des ressources en vue d'atteindre un objectif." }\end{array}$ \\
\hline $\begin{array}{l}\text { Marchesnay } \\
\text { (2004) }\end{array}$ & $\begin{array}{l}\text { "L'ensemble constitué par les réflexions, les décisions, les actions ayant pour objet } \\
\text { de déterminer les buts généraux, puis les objectifs, de fixer le choix des moyens } \\
\text { pour réaliser ces buts, de mettre en cuvre les actions et les activités en } \\
\text { conséquence, de contrôler les performances attachées à cette exécution et à la } \\
\text { réalisation des buts." }\end{array}$ \\
\hline $\begin{array}{l}\text { Ardoin et al., } \\
(2009)\end{array}$ & $\begin{array}{l}\text { "Pour une entreprise, la stratégie consiste à choisir ses activités et à allouer ses } \\
\text { ressources de manière à atteindre un niveau de performance durablement } \\
\text { supérieur à celui de ses concurrents dans ces activités, dans le but de créer de la } \\
\text { valeur pour ses actionnaires." }\end{array}$ \\
\hline
\end{tabular}

9 À travers la stratégie, l'entreprise va déterminer ses buts et ses objectifs. Le management stratégique implique aussi des actions et des choix quant au déploiement et à l'allocation des ressources. Desreumaux et al. (2009) résument que la stratégie consiste à identifier le pourquoi (1), le quoi (2) et le comment (3) de l'activité de l'entreprise :

(1) Le pourquoi correspond à la mission et aux ambitions à long terme de l'entreprise ;

(2) Le quoi illustre les buts ${ }^{5}$ et les objectifs ${ }^{6}$ de l'entreprise ;

(3) Le comment définit les choix et les actions pour atteindre les buts et les objectifs.

10 La création de valeur, l'affrontement concurrentiel et le choix du périmètre des activités sont également des dimensions fondamentales du management stratégique (Ardoin et al., 2009). La stratégie requiert un ajustement perpétuel aux menaces et aux opportunités ${ }^{7}$ de l'environnement de l'entreprise de plus en plus fluctuant et incertain. Les institutions y jouent également un rôle majeur en contraignant les pratiques des organisations. Outre la conquête des différents marchés liés à son activité, l'entreprise doit adopter des conduites socialement acceptables (Koenig, 2004). Pour cet auteur, l'entreprise est confrontée à trois impératifs stratégiques pour assurer sa pérennité dans le temps: la compétitivité, la sécurité et la légitimité (tableau 2).

Tableau 2. Les buts stratégiques d'une organisation

\begin{tabular}{|l|l|l|}
\hline Compétitivité & $\underline{\text { Sécurité }}$ & $\underline{\text { Légitimité }}^{8}$ \\
\hline
\end{tabular}




\begin{tabular}{|l|ll|l|l|}
\hline $\begin{array}{l}\text { Assurer sa capacité à } \\
\text { créer et conserver } \\
\text { une clientèle }\end{array}$ & $\begin{array}{l}\text { Assurer son existence et } \\
\text { anticiper les risques liés à }\end{array}$ & $\begin{array}{l}\text { Assurer l'obtention du «droit } \\
\text { d'exercer son activité » en } \\
\text { l'activité économique et aux } \\
\text { décisions stratégiques }\end{array}$ & $\begin{array}{l}\text { aunt attentes de ses } \\
\text { parties prenantes internes et } \\
\text { externes }\end{array}$ \\
\hline
\end{tabular}

Source : Koenig, 2004

11 L'écologie industrielle requiert l'existence d'un contexte propice à la collaboration (Korhonen, 2004 ; Buclet, 2009). Le partenariat, les stratégies collectives et les réseaux inter-organisationnels font l'objet d'une littérature abondante en management stratégique qui pourrait enrichir les travaux sur l'écologie industrielle.

12 Si les expériences d'écologie industrielle semblent se multiplier sur le terrain ${ }^{9}$, la majorité des pratiques relève de la mutualisation ${ }^{10}$. La substitution, qui consiste à remplacer les matières premières traditionnelles par des déchets, peine à trouver un écho opérationnel. Obligeant l'entreprise à sortir de la gestion quotidienne de son activité pour considérer ses flux matériels et énergétiques tout en impliquant la création de partenariats, l'écologie industrielle est résolument complexe à développer. Elle constitue, à notre sens, un objet de recherche intéressant pour le management stratégique. Pour favoriser son développement opérationnel, il semble nécessaire d'analyser et de comprendre ses implications stratégiques et organisationnelles pour l'entreprise.

\section{Les principaux bénéfices et menaces de l'écologie industrielle pour l'entreprise}

13 L'écologie industrielle intéresse la communauté académique depuis les années 1990. Cet engouement a donné lieu à la production de nombreux écrits. Un examen de la littérature permet la mise en évidence de ses problématiques opérationnelles pour l'organisation (bénéfices et menaces).

14 L'écologie industrielle permettrait de sortir du débat «Économie versus Écologie » (Sterr et Ott, 2004; Erkman, 2004). Elle peut aussi contribuer à l'atteinte des trois buts stratégiques de l'organisation définis par Koenig (2004). Le tableau 3 reprend les principaux bénéfices de l'écologie industrielle au regard de cette typologie.

Tableau 3. Les bénéfices issus des pratiques éco-industrielles au regard de la taxonomie de Koenig (2004)

\begin{tabular}{|l|l|l|}
\hline Compétitivité & Sécurité & Légitimité \\
\hline $\begin{array}{l}\text { Revenus supplémentaires } \\
\text { Coûts moindres }\end{array}$ & $\begin{array}{l}\text { Réorientation d'une activité menacée } \\
\text { Nouveaux marchés } \\
\text { Coopération interentreprises sécurisé à des ressources critiques }\end{array}$ & Image de marque \\
\hline
\end{tabular}

15 La compétitivité : L'écologie industrielle peut conférer une certaine valeur au déchet (Korhonen et al., 2004), traditionnellement perçu comme une source de coûts liés à son 
traitement et à son élimination obligatoires (Lavoillotte, 2006). La commercialisation de sous-produits crée alors des revenus supplémentaires pour l'entreprise qui peut aussi voir certains coûts diminuer (collecte, traitement et/ou élimination). Dans un contexte global de raréfaction des ressources induisant fatalement une augmentation durable de leur coût d'achat (Jurgensen, 2009), le développement des synergies de substitution peut permettre à l'entreprise d'échapper à des coûts d'approvisionnement excessifs et rester compétitive (Cosgriff Dunn et Steinemann, 1998). La valorisation des déchets permet également d'aborder de nouveaux marchés et de diversifier le portefeuille de clients. Enfin, l'écologie industrielle permet la mise en relation d'acteurs habituellement peu amenés à coopérer (Buclet, 2009) favorisant notamment les partages d'expérience et les transferts de bonnes pratiques.

La sécurité : L'écologie industrielle offre des pistes de réflexion pour les entreprises dont l'activité est menacée (cf. le cas de la distillerie Dislaub présenté par Schalchli [2008]). Par ailleurs, une démarche éco-industrielle peut permettre de sécuriser l'accès des acteurs à des ressources critiques telles que l'eau, l'énergie et les matières premières (Chertow et Lombardi, 2005).

17 La légitimité : L'écologie industrielle constitue une démarche responsable susceptible de différencier l'entreprise et d'améliorer son image de marque. Or, les actifs intangibles et particulièrement la marque, revêtent une importance croissante (Legendre et Coderre, 2008). L'entreprise qui adopte des pratiques éco-industrielles devrait être valorisée, notamment par les consommateurs occidentaux qui affichent un intérêt grandissant envers les produits respectueux de l'environnement (Laville et Deveaux, 2008).

18 Certains travaux ont aussi souligné les menaces de l'écologie industrielle pour l'organisation (tableau 4).

Tableau 4. Les menaces issues des pratiques éco-industrielles

\begin{tabular}{|l|}
\hline Menaces de l'écologie industrielle \\
\hline Légitimité des pratiques éco-industrielles \\
Variabilité des flux et inflexibilité des process \\
Dépendance des acteurs \\
Immobilisme technologique \\
Complexité organisationnelle \\
Opportunisme de l'entreprise \\
\hline
\end{tabular}

La valorisation des déchets peut déplaire à certaines parties prenantes de l'entreprise (ex: les riverains, les salariés, les associations de protection de l'environnement, etc.) (Adoue, 2007 ; Schalchli, 2008). Ce syndrome NIMBY pour "Not In My BackYard" (en français : "Pas Dans Mon Jardin ${ }^{11}$ ) peut se traduire par des réactions négatives du public pouvant menacer la pérennité de l'entreprise (boycott, dévalorisation de l'image de l'entreprise, arrêt ou non-réalisation des synergies éco-industrielles, retrait du «droit d'exercer son activité »). La rigidité des procédés industriels (Erkman, 2002 ; Buclet, 2011) et les variations des flux de matières résiduelles (qualité, quantité, composition, taille) peuvent poser des difficultés aux organisations qui exploitent ces ressources secondaires ${ }^{12}$. Les entreprises doivent apprendre à maîtriser les flux de sous-produits qui différent 
des approvisionnements traditionnels (Boiral et Kabongo, 2004). La stabilité des écosystèmes naturels repose partiellement sur l'hypothèse de la redondance-résilience ${ }^{13}$ (Côté et Smolenaars, 1997; Erkman, 2004; Zhu et Côté, 2004 ; Pelt et al., 2010). Or la spécificité de chaque synergie de substitution peut diminuer le nombre de fournisseurs potentiels (Sterr et Ott, 2004). Une forte dépendance peut s'instaurer entre les partenaires rendant l'écosystème industriel vulnérable aux pressions internes et externes (Ehrenfeld et Gertler, 1997 ; Cosgriff Dunn et Steinemann, 1998 ; Van Leeuwen et al., 2003 ; Tranchant et al., 2004 ; Korhonen et al., 2004 ; Schalchli, 2008). Une symbiose industrielle peut disparaître si l'un des partenaires stoppe ou délocalise son activité, ou même s'il modifie simplement ses procédés (Sterr et Ott, 2004). Les pratiques éco-industrielles peuvent donc engendrer un risque d'inertie technologique pour les partenaires à travers le maintien de procédés obsolètes et peu efficaces sur le plan environnemental (Chertow, 2007 ; Schalchli, 2008). Les industriels peuvent être tentés de conserver leurs process tels quels pour continuer à échanger leurs sous-produits (Korhonen et al., 2004). L'écologie industrielle est donc susceptible de freiner l'innovation (Tranchant et al., 2004) car un changement chez l'un des partenaires pourrait «briser» la stabilité de la symbiose industrielle. Les autres acteurs seraient alors contraints d'adapter leur procédé et/ou sous-produits pour poursuivre les échanges ${ }^{14}$. L'imbrication des procédés industriels génère une complexité organisationnelle susceptible de diminuer la flexibilité des entreprises (Van Leeuwen et al., 2003) et d'augmenter les risques de conflits d'intérêts. Enfin, le bouclage des flux n'est pas toujours souhaitable (cf. crise de la "vache folle » [Erkman, 2005 ; Adoue, 2007]). Motivés par l'appât du gain, les industriels peuvent être tentés de recycler des matières dont la valorisation est interdite ou peut être dangereuse ; voire de ne pas chercher à diminuer leur production de déchets si la synergie de substitution s'avère rentable (Cosgriff Dunn et Steinemann, 1998). L'écologie industrielle ne doit donc pas être une stratégie visant à valoriser imprudemment tous les déchets.

En substance, ces problématiques opérationnelles (bénéfices et menaces) révèlent trois enjeux stratégiques de l'écologie industrielle pour l'entreprise: légitimité et responsabilité sociétale, relations inter-organisationnelles et performance et avantage concurrentiel (tableau 5).

Tableau 5. Les enjeux stratégiques de l'écologie industrielle pour l'entreprise

\begin{tabular}{|c|c|c|}
\hline $\begin{array}{l}\text { Bénéfices de l'écologie } \\
\text { industrielle }\end{array}$ & $\begin{array}{l}\text { Menaces de l'écologie } \\
\text { industrielle }\end{array}$ & $\begin{array}{l}\text { Enjeux stratégiques pour } \\
\text { 1'entreprise }\end{array}$ \\
\hline Image de marque & $\begin{array}{l}\text { Légitimité des pratiques } \\
\text { éco-industrielles } \\
\text { Opportunisme } \\
\text { l'entreprise }\end{array}$ & $\begin{array}{l}\text { Légitimité } \\
\text { responsabilité sociétale }\end{array}$ \\
\hline $\begin{array}{r}\text { Coopération } \\
\text { interentreprises }\end{array}$ & $\begin{array}{l}\text { Dépendance des acteurs } \\
\text { Immobilisme } \\
\text { technologique } \\
\text { Complexité } \\
\text { organisationnelle }\end{array}$ & $\begin{array}{l}\text { Relations } \\
\text { organisationnelles }\end{array}$ \\
\hline $\begin{array}{l}\text { Coûts moindres } \\
\text { Revenus supplémentaires } \\
\text { Nouveaux marchés } \\
\text { Image de marque } \\
\text { Réorientation } \\
\text { activité menacée d'une } \\
\text { Accès sécurisé à des } \\
\text { ressources critiques }\end{array}$ & $\begin{array}{l}\text { Variabilité des flux et } \\
\text { inflexibilité des process }\end{array}$ & $\begin{array}{l}\text { Performance et avantage } \\
\text { concurrentiel }\end{array}$ \\
\hline
\end{tabular}




\section{L'écologie industrielle et le management stratégique : enjeux, perspectives et orientations de recherche}

La littérature met donc en lumière certaines problématiques opérationnelles de l'écologie industrielle, notamment ses avantages et ses inconvénients pour l'entreprise. À partir de ces éléments et d'autres questionnements, nous esquissons un agenda de recherche en mobilisant le champ du management stratégique. Nos propositions s'appuient sur différents cadres conceptuels permettant l'étude des pratiques d'écologie industrielle sous différents angles. À cet égard, Maxwell (1999 : 67) emprunte la métaphore de la "théorie comme porte-manteau» à Jane Margolis: "Les concepts des théories existantes constituent le "porte-manteau" du placard; ils offrent des endroits où "accrocher" les données en montrant leurs liens avec d'autres données. Toutefois, aucune théorie n'intégrera toutes les données de façon également satisfaisante». Dès lors, la multiplication des cadres conceptuels parait indispensable pour appréhender un phénomène complexe et multidimensionnel tel que l'écologie industrielle.

À chaque enjeu stratégique regroupant un ensemble de problématiques opérationnelles, nous associons des grilles de lecture exploitables dans une perspective stratégique :

- Les questions portant sur la légitimité et la responsabilité de l'organisation peuvent être analysées à la lueur de la théorie des parties prenantes ou du néo-institutionnalisme sociologique ;

- Les problématiques relatives aux relations inter-organisationnelles peuvent être examinées au moyen de l'économie des coûts de transaction ou de l'approche relationnelle;

- Les enjeux de performance et d'avantage concurrentiel peuvent être étudiés à l'aide du business model ou de la théorie des ressources.

Avant de présenter les cadres conceptuels et les pistes de recherche sur l'écologie industrielle associées, nous ouvrons une parenthèse sur les deux grandes orientations que peut prendre une recherche en management. En effet, un objet peut être étudié par son contenu mais aussi par son processus (tableau 6).

Tableau 6. Les recherches « contenu » et « processus »

\begin{tabular}{|l|l|l|}
\hline & Approche « contenu » & Approche «processus » \\
\hline & Composition de l'objet & Comportement de l'objet dans le temps \\
\hline $\begin{array}{l}\text { Description de } \\
\text { l'objet }\end{array}$ & Statique & Dynamique \\
\hline $\begin{array}{l}\text { Exemple de } \\
\text { question } \\
\underline{\text { recherche }}\end{array}$ & $\begin{array}{l}\text { "Comment expliquer le contrôle } \\
\text { exercé par certaines entreprises } \\
\text { sur d'autres au sein d'un réseau?» }\end{array}$ & $\begin{array}{l}\text { "Comment naissent des accords inter- } \\
\text { organisationnels et comment se } \\
\text { structurent-ils dans le temps? » }\end{array}$ \\
\hline
\end{tabular}


24 les études « processus » à saisir et retracer un phénomène et son évolution dans le temps (Grenier et Josserand, 2007). Dans notre perspective, une approche « contenu » pourrait par exemple s'intéresser à la nature des relations entre les membres d'un écosystème industriel. Une orientation "processus » pourrait quant à elle, s'interroger sur la construction et l'évolution des rapports entre les membres d'une symbiose industrielle.

\subsection{La légitimité et la responsabilité sociétale de l'entreprise}

La théorie des parties prenantes (Freeman, 1984) s'oppose à la conception actionnariale ${ }^{15}$ de Friedman (1970). Les questions de Responsabilité Sociétale de l'Entreprise (RSE) et de gouvernance invitent l'organisation à reconnaître et prendre en compte les externalités de ses activités. Sommairement, la RSE revient à se poser la question suivante : « de quoi l'entreprise est-elle responsable et devant qui ?» (Cazal, 2008: 15). La RSE concerne donc les parties prenantes ${ }^{16}$ de l'entreprise qui sont les individus ou les groupes qui peuvent affecter ou sont affectés par ses actions (Freeman, 1984). La légitimité ${ }^{17}$ auprès de ces acteurs est l'un des buts stratégiques de l'entreprise (Koenig, 2004). Mais d'une entreprise à une autre, les parties prenantes et leur influence varient. À cet égard, Mendelow (1991) propose une matrice pour évaluer leur importance (tableau 7).

Tableau 7. La matrice « intérêt-pouvoir » des parties prenantes de Mendelow (1991)

\begin{tabular}{|l|l|l|l|}
\hline \multicolumn{2}{|l|}{} & \multicolumn{2}{l|}{$\begin{array}{l}\text { Niveau d'intérêt de la partie } \\
\text { prenante }\end{array}$} \\
\cline { 3 - 4 } & & Faible & Élevé \\
\hline \multirow{2}{*}{ Pouvoir de la partie prenante } & Faible & Effort minimal & À informer \\
\cline { 2 - 4 } & Élevé & À satisfaire & Joueurs clés \\
\hline
\end{tabular}

Le pouvoir de la partie prenante est évalué en fonction de sa capacité à influencer la réalisation des projets de l'organisation. Le niveau d'intérêt de la partie prenante vis-à-vis de l'entreprise doit également être apprécié.

Le néo-institutionnalisme sociologique (Meyer et Rowan, 1977 ; DiMaggio et Powell, 1983) vise à comprendre le changement organisationnel et l'isomorphisme interorganisationnel. Trois formes de pressions influencent le comportement des organisations et peuvent expliquer l'adoption de nouvelles pratiques: coercitives ${ }^{18}$, mimétiques ${ }^{19}$ et normatives ${ }^{20}$ (DiMaggio et Powell, 1983). Dans les faits, «ces trois processus se combinent et il est difficile d'associer un changement à une forme unique de pression.» précisent néanmoins Ardoin et al. (2009: 757). Les organisations agiraient ainsi plus pour se conformer à des pressions institutionnelles et pour éviter les risques que pour atteindre une efficacité optimale (Meyer et Rowan, 1977 ; DiMaggio et Powell, 1983). À la recherche de légitimité sociale, les entreprises imitent «des pratiques qui semblent réussir ailleurs et/ou qui sont valorisées dans la communauté à laquelle elles appartiennent. » (Leca, $2006: 69)$. 

étudiées par la théorie des parties prenantes et le néo-institutionnalisme sociologique.

Tableau 8. Écologie industrielle, légitimité et responsabilité sociétale

\begin{tabular}{|c|c|}
\hline Cadres conceptuels & $\begin{array}{l}\text { Problématiques de l'écologie } \\
\text { industrielle }\end{array}$ \\
\hline $\begin{array}{l}\text { Théorie des parties prenantes: responsabilité } \\
\text { sociétale, éthique, parties prenantes }\end{array}$ & $\begin{array}{l}\text { Légitimité de l'organisation et satisfaction } \\
\text { des parties prenantes; }\end{array}$ \\
\hline $\begin{array}{l}\text { Néo-institutionnalisme sociologique } \\
\text { sociale, isomorphisme, institutions }\end{array}$ & $\begin{array}{l}\text { Légitimité des pratiques }^{21} \text {; } \\
\text { Motivations des pratiques; } \\
\text { Résultats (image de marque, notoriété, } \\
\text { réputation); } \\
\text { Problématiques éthiques }^{22} \text {; } \\
\text { Influence des premières expériences }{ }^{23} \\
\text { Perspectives et limites du mimétisme }{ }^{24} \\
\text { (spécificité des territoires) }^{25} \text {. }\end{array}$ \\
\hline
\end{tabular}

\subsection{Les relations inter-organisationnelles}

Issue des travaux de Coase (1937) et Williamson $(1975,1985,1996)$, la théorie des coûts de transaction considère que toute transaction entre deux partenaires engendre des coûts spécifiques. Ces coûts de transaction peuvent être ex ante (recherche et sélection du partenaire, négociation et rédaction du contrat) ou ex post (surveillance et contrôle, renégociation ou rupture du contrat) (Williamson, 1985). Plusieurs facteurs sont à l'origine de ces coûts de transaction : le comportement des acteurs (rationalité limitée ${ }^{26}$ et opportunisme ${ }^{27}$ ) et la nature des transactions (spécificité des actifs ${ }^{28}$, incertitude ${ }^{29}$ et fréquence ${ }^{30}$ ). L'évaluation des coûts et des risques induits par la transaction doit amener l'entreprise à opter pour "la structure de gouvernance la plus économique » (Williamson, 1979: 235) : le marché ou la firme.

Contrairement à Porter (1980) qui considère que les relations interentreprises reposent uniquement sur la rivalité, l'approche relationnelle de Dyer et Singh (1998) entrevoit la coopération et les alliances comme un moyen d'augmenter les performances des organisations. Dans cette perspective, l'avantage concurrentiel durable provient des relations partenariales conclues entre les firmes. La coopération interentreprises permet la création de rentes relationnelles ${ }^{31}$ issues des investissements dans des actifs spécifiques, du partage de connaissances, de la mise en commun de ressources complémentaires ou encore du choix d'une gouvernance efficace.

31 Le tableau 9 recense les problématiques de l'écologie industrielle susceptibles d'être étudiées par la théorie des coûts de transaction et l'approche relationnelle.

Tableau 9. Écologie industrielle et relations inter-organisationnelles

\begin{tabular}{|l|l|}
\hline Cadres conceptuels & Problématiques de l'écologie industrielle \\
\hline
\end{tabular}




\begin{tabular}{|l|l|}
\hline $\begin{array}{l}\text { Théorie des coûts de transaction : } \\
\text { opportunisme, coordination, relations } \\
\text { inter-organisationnelles, coûts de } \\
\text { transaction }\end{array}$ & $\begin{array}{l}\text { Coûts de transaction; } \\
\text { Coordination des partenaires (confiance, degré de } \\
\text { formalisation des accords et contractualisation); } \\
\text { Synergies et modes de coordination retenus ; } \\
\text { Dépendance et opportunisme (pouvoir de } \\
\text { négociation, investissements non redéployables, coûts } \\
\text { de transfert, incertitude, immobilisme technologique, } \\
\text { complexité organisationnelle); } \\
\text { Approche relationnelle : } \\
\begin{array}{l}\text { coopération interentreprises, rentes d'un acteurs-tiers }{ }^{32} ; \\
\text { relationnelles, performance }\end{array}\end{array}$ \\
$\begin{array}{l}\text { Performance des partenaires (routines partagées, } \\
\text { langage commun, nouvelles compétences); } \\
\text { Synergies et rentes relationnelles (nature et } \\
\text { répartition entre les entreprises); } \\
\text { Innovation (partage d'informations et de savoir- } \\
\text { faire). }\end{array}$ \\
\hline
\end{tabular}

\subsection{La performance et l'avantage concurrentiel}

Lecocq et al. (2006:5) définissent le business model ${ }^{33}$ comme "les choix qu'une entreprise effectue pour générer des revenus». Le business model ne décrit pas les missions de l'entreprise, mais il détermine comment les remplir pour créer de la valeur (Lecocq et al., 2004 ; Teece, 2010). Lehmann-Ortega (2008) propose trois composantes pour décrire un business model : la proposition de valeur et l'équation de profit (figure 1).

Figure 1. Les trois composantes du Business Model

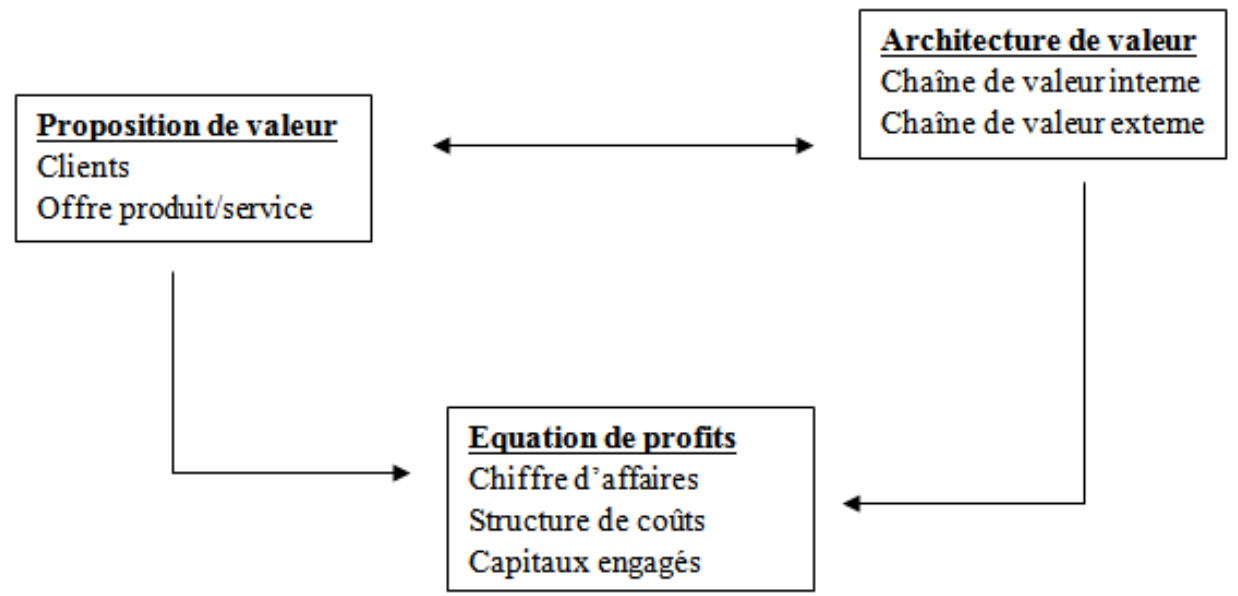

Source : Lehmann-Ortega (2008)

À partir de ses revenus et de ses coûts, le business model doit générer des profits pour l'entreprise tout en rémunérant les capitaux engagés. Le business model n'est pas immuable. Il doit évoluer pour s'adapter aux fluctuations de l'environnement afin d'assurer la pérennité de l'organisation sur le marché. Plébiscité pour son caractère opérationnel, le business model est une source de créativité pour l'entreprise (Lecocq et 


\section{Conclusion} prenantes ou le néo-institutionnalisme sociologique. La théorie des coûts de transaction ou l'approche relationnelle pourraient faciliter l'analyse des relations interorganisationnelles. Enfin, les problématiques liées à la performance des firmes trouveraient un écho à travers le business model ou la théorie des ressources.

41 Si cette contribution vise principalement les chercheurs, l'objectif du management stratégique reste de réaliser des apports managériaux. Desreumaux (2008:68-69) rappelle en effet que "le management stratégique n'est pas un champ uniquement théorique ou académique mais se doit d'apporter une contribution à la résolution des problèmes que rencontrent, d'un point de vue stratégique, les organisations de toute catégorie, de toute taille et de toute localisation. » Ainsi, la conduite de recherches sur l'écologie industrielle, dans la perspective du management stratégique, permettrait de participer à l'élaboration de recommandations managériales utiles aux praticiens. 


\section{BIBLIOGRAPHIE}

Adoue C., 2007 ( 1 ère édition), Mettre en œuvre l'écologie industrielle, Lausanne, Presses polytechniques et universitaires romandes, collection «Sciences et ingénierie de l'environnement ».

Allenby B.R., 1992, Design for Environment : Implementing Industrial Ecology, Thèse de doctorat, Sciences de l'Environnement, Université du New Jersey.

Ardoin J.-L., Barbier E., Blanc G., Bordès-Pagès, G., Capron L., Castañer X., Crémadez M., Détrie J.P., Durand R., Dussauge P., Faugère M., Garrette B., Gottschalg O., Iselin F., Larçon J.-P., Laroche H., Lehmann-Ortega L., Le Joly K., Moatti V., Moingeon B., Nioche J.- P., Quélin B., Quémard E., Ramanantsoa B., Reitter R., Santi M., Soenen G., Toche V., 2009 (5 ${ }^{\text {ème }}$ édition), Strategor, Paris, Dunod, collection « Livres en Or ».

Ayres R.U., 1989, « Le métabolisme industriel et les changements de l'environnement planétaire », Revue internationale de sciences sociales, n 121, p. 401-412.

Baas L., 2007, "Industrial Ecology as Regional Corporate Sustainability System", $14^{\text {th }}$ GIN Conference Sustainable Ecosystem and Social Stewardship, Waterloo (Canada), du 15 au 17 juin, 27 p.

Barney J.B., 1991, "Firm resources and sustained competitive advantage", Journal of Management, vol. 17, p. 99-120.

Bent K., 1990, La Stratégie des Affaires, Les Presses du Management.

Bey C., 2008, «L'écologie industrielle : promesses et limites d'une approche pratique du management durable en entreprise », in Dion, M., Wolff, D. (dir.), Le développement durable Théories et applications au management, Paris, Dunod, collection « Stratégie et politique d'entreprise ", p. 109-122.

Boiral O., Kabongo J., 2004, « Le management des savoirs au service de l'écologie industrielle », Revue française de gestion, $\mathrm{n}^{\circ} 149$, p. 173-191.

Boons F., Baas L., 2006, "Industrial symbiosis in a social science perspective" in Lombardi, R. and Laybourn P. (dirs.), Industrial Symbiosis in Action, Yale School of Forestry \& Environmental Studies, Report $n,{ }^{\circ} 12$, p. $77-82$.

Bouba-Olga O., 2003, L'économie de l'entreprise, Paris, éditions du Seuil, collection « Économie ».

Brent A. C., Oelofse S., Godfrey L., 2008,"Advancing the concepts of industrial ecology in South African institutions" South African Journal of Science, $\mathrm{n}^{\circ}$ 104, January/February, p. 9-12.

Brown L.R., 2003 (1 1 ère édition), Eco-économie. Une autre croissance est possible, écologique et durable, Paris, éditions du Seuil, collection « Économie humaine ».

Buclet N., 2009, Les déclinaisons territoriales des stratégies de développement durable : à la recherche de l'espace-temps perdu, mémoire préparé pour soutenir une Habilitation à Diriger des Recherches, Aménagement, Université de technologies de Troyes.

Buclet N., 2011, Écologie industrielle et territoriale - stratégies locales pour un développement durable, Presses Universitaires du Septentrion, Villeneuve d'Ascq. 
Cazal D., 2008, « Parties prenantes et RSE : des enjeux sociopolitiques au-delà des contrats », Revue de l'organisation responsable, vol. 3, $\mathrm{n}^{\circ}$ 1, p. 12-23.

Chandler A. D., 1962, Stratégies et structures de l'entreprise, Éditions d'Organisation.

Chertow M.R., Lombardi R., 2005, "Quantifying Economic and Environmental Benefits of CoLocated Firms", Environmental Science \& Technology, vol. 39, n 17, p. 6535-6541.

Chertow M., 2007, "Industrial Symbiosis" Encyclopedia of Earth, Eds. Cutler J. Cleveland (Washington, D.C.: Environmental Information Coalition, National Council for Science and the Environment), [En ligne], consulté le 09 janvier 2012. URL : http://www.eoearth.org/article/ Industrial_symbiosis.

Coase R.H., 1937, "The nature of the firm", Economica, vol. 4, p. 386-405.

Cosgriff Dunn B., Steinemann A., 1998, "Industrial Ecology for Sustainable Communities", Journal of Environmental Planning and Management, vol. 41, Issue 6, p. 661-672.

Côté R., Smolenaars T., 1997, "Supporting pillars for industrial ecosystems", Journal of Cleaner Production, vol. 5 Issues 1-2, p. 67-74.

Desreumaux A., 2008, « Refaire de la stratégie? », Finance Contrôle Stratégie, vol. 11, hors-série, juin, p. 67-107.

Desreumaux A., Lecocq X., Warnier V., 2009 ( 2 ème édition), Stratégie, Paris, Pearson Education, collection « Synthex ».

DiMaggio P., Powell W., 1983, "The iron cage revisited : institutional isomorphism and collective rationality in organizational fields", American Sociological Review, vol. 48, Issue 2, p. 147-160.

Dyer J.H., Singh H., 1998, "The relational view: cooperative strategy and sources of interorganizational competitive advantage", Academy of Management Review, vol. 23, $\mathrm{n}^{\circ} 4$, p. 660-679.

Ehrenfeld J.R., Gertler N., 1997, "Industrial Ecology in Practice - The Evolution of Interdependence at Kalundborg", Journal of Industrial Ecology, vol. 1, Issue 1, p. 67-79.

Erkman S., 2002, « Les applications de l'écologie industrielle », Les Cahiers français, n 306, dossier « enjeux et politiques de l'environnement » réalisé sous la direction de Philippe Tronquoy, p. 50-51.

Erkman S., 2004 ( $2^{\text {ème }}$ édition), Vers une écologie industrielle. Comment mettre en pratique le développement durable dans une société hyper-industrielle, Paris, Charles Léopold Mayer, collection « Dossiers pour un débat ».

Erkman S, 2005, «L'écologie industrielle, entretien avec Suren Erkman », in Allemand, S. (dir.), Les nouveaux utopistes de l'économie Produire, consommer, fabriquer... différemment, Paris, Autrement, collection « Frontières"

Freeman R. E., 1984 (1 1 ère edition), Strategic Management: A Stakeholder Approach, Boston, Pitman.

Freeman R.E., 1999, "Response: Divergent Stakeholder Theory", Academy of Management Review, vol. $24, n^{\circ} 2$, p. 233-236.

Friedman M., 1970, "The Social Responsibility of Business is to Increase its Profits", The New York Times Magazine, September 13.

Frosch R. A., Gallopoulos N. E., 1989, "Strategies for Manufacturing", Scientific American, 261 : 3, Special Issue : Managing Planet Earth, p. 144-152. 
Frosch R. A., 1992, "Industrial ecology: A philosophical introduction", Proc. Nati. Acad. Sci. USA, vol. 89, p. 800-803.

Grenier C., Josserand E., 2007 (3 $3^{\text {ème }}$ édition), « Recherches sur le contenu et recherches sur le processus » in Thiétart, R.-A. (dir.), Méthodes de recherche en management, Paris, Dunod, collection «Management et Ressources Humaines ».

Jurgensen P., 2009, L'économie verte - comment sauver notre planète, Odile Jacob.

Koenig G., 2004 (2 2 ème édition), Management stratégique. Projets, interactions et contextes, Paris, Dunod, collection « Stratégie et politique d'entreprise ».

Korhonen J., 2004, "Industrial ecology in the strategic sustainable development model: strategic applications of industrial ecology", Journal of Cleaner Production, vol. 12, p. 809-823.

Korhonen J., Malmborg F., Von Malmborg F., Strachan P., Ehrenfeld, J., 2004, "Management and policy aspects of Industrial Ecology: An emerging research agenda". Business Strategy and the Environment, vol. 13, Issue 5, p. 289-305.

Labourdette A., 1989, Stratégies d'Entreprise, Montchrestien.

Laville E., Deveaux R., 2008, « La "nouvelle frontière" du marketing responsable » in : Dion M., Wolff D. (dir.), Le développement durable - Théories et applications au management, Paris, Dunod, collection « Stratégie et politique d'entreprise », p. 189-207.

Lavoillotte M.-P., 2006, « Propriété et technique contractuelle au service de la gestion des déchets », in Falque, M., Lamotte, H., Saglio, J.-F. (dir.). Les déchets. Droit de propriété, Économie et Environnement, Bruxelles, Bruylant, p. 111-122.

Leca B., 2006, « Pas seulement des "lemmings". Les relations entre les organisations et leur environnement dans le néo-institutionnalisme sociologique », Finance Contrôle Stratégie, vol. 9, n ${ }^{\circ} 4$, décembre, p. 67-86.

Lecocq X., Demil B., Warnier V., 2004, « Le business model : l'oublié de la stratégie », $13^{\text {ème }}$ Conférence Internationale de Management Stratégique, Normandie, Vallée de Seine (France), juin, du 2 au 4 juin.

Lecocq X., Demil B., Warnier V., 2006, « Le business model, un outil d'analyse stratégique », L'Expansion Management Review, $\mathrm{n}^{\circ}$ 123, p. 96-109.

Legendre, S., Coderre, F., 2008, « Rapports annuels, responsabilité sociale de l'entreprise et valorisation de la marque ", in : Dion, M., Wolff, D. (dir.), Le développement durable - Théories et applications au management, Paris, Dunod, collection « Stratégie et politique d'entreprise », p. 173-187.

Lehmann-Ortega, L., 2008, "Business Model: from buzz word to managerial tool", Working Paper.

Maxwell J.A., 1999 (1 1 ère édition), La modélisation de la recherche qualitative. Une approche interactive, Academic Press Fribourg, Suisse.

Marchesnay M., 2004, Management stratégique, [En ligne], consulté le 12 septembre 2012. URL : http://asso.nordnet.fr/adreg/Adreg_08_MM.pdf.

Mendelow A., 1991, Proceedings of the Second International Conference on Information Systems, Cambridge (MA).

Meyer J.W., Rowan B., 1977, "Institutionalized Organizations: Formal Structure as Myth and Ceremony", American Journal of Sociology, vol. 83, n² 2, p. 340-363.

Pelt J.-M., Cheissoux D., Steffan F., 2010, Les dons précieux de la nature, Paris, Fayard. 
Penrose E.T., 1959 (1 $1^{\text {ère }}$ edition), The Theory of the Growth of the Firm, Wiley.

Porter, M.E., 1980 (1 1 ère edition), Competitive Strategy : Techniques for Analyzing Industries and Competitors, The Free Press.

Schalchli P., 2008, Mettre en œuvre une démarche d'écologie industrielle sur un parc d'activité, Lyon, éditions DPE, Collection Orée, Environnement.

Simon H., 1961 (2 ème édition), Administrative Behaviour, New York, MacMillan.

Soparnot, R., Grandval, S., 2008, « Intégrer le développement durable dans le business model de l'entreprise ", in Dion, M., Wolff, D. (dir.), Le développement durable - Théories et applications au management, Paris, Dunod, collection « Stratégie et politique d'entreprise », p. 123-137.

Sterr T., Ott T., 2004, "The industrial region as a promising unit for eco-industrial development reflections, practical experience and establishment of innovative instruments to support industrial ecology", Journal of Cleaner Production, vol. 12, p. 947-965.

Teece D., 2010, "Business Models, Business Strategy and Innovation", Long Range Planning, vol. 43, Issues 2-3, p. 172-194.

Thiétart R.-A., 1990, La Stratégie d'Entreprise, McGraw-Hill.

Tranchant C., Vasseur L., Ouattara I., Vanderlinden J.-P., 2004, «L'écologie industrielle : une approche écosystémique pour le développement durable », colloque international sur le Développement Durable, Ouagadougou (Burkina Faso), du $1^{\mathrm{er}}$ au 4 juin.

Van Leeuwen M.G., Vermeulen W.J.V., Glasbergen P., 2003, "Planning eco-industrial parks: an analysis of Dutch planning methods", Business Strategy and the Environment, vol. 12, Issue 3, p. $147-162$.

Von Neumann J., Morgenstern O., 1947, Theory of Games and Economic Behavior, Princeton University Press.

Weppe X., Warnier V., Lecocq X., 2012, «Ressources stratégiques, ressources ordinaires et ressources négatives : Pour une reconnaissance de l'ensemble du spectre des ressources », Working Paper.

Williamson O.E., 1975 (1 1 ère edition), Market and Hierarchies: Analysis and Antitrust Implications. A Study in the Economics of Internal Organization, New York, Free Press.

Williamson O.E., 1979, "Transaction-cost Economics: The Governance of Contractual Relations", Journal of Law and Economics, XXII (2), October, p. 233-261.

Williamson O.E., 1985 ( $1^{\text {ère }}$ edition), The Economic Institutions of Capitalism, New York, Free Press.

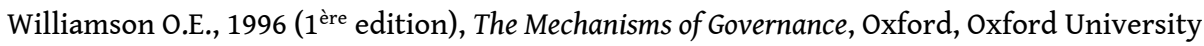
Press.

Zhu Q., Côté R.P., 2004, "Integrating green supply chain management into an embryonic ecoindustrial development: a case study of the Guitang Group", Journal of Cleaner Production, vol. 12, Issues 8-10, p. 1025-1035.

\section{NOTES}

1. C'est un article de Frosch et Gallopoulos (1989) qui catalyse le débat sur l'écologie industrielle. Leur contribution appelle les industriels à développer des méthodes de production plus respectueuses de l'environnement en s'inspirant des écosystèmes naturels. S'en suivent: la 
découverte de la symbiose de Kalundborg, la création de revues spécialisées, la constitution d'une communauté académique, etc.

2. La loi n75-633 du 15 juillet 1975 définit le déchet comme "tout résidu d'un processus de production, de transformation ou d'utilisation, de toute substance, matériau, produit ou plus généralement tout bien meuble abandonné ou que son détenteur destine à l'abandon ».

3. Les écosystèmes naturels sont constitués de chaînes alimentaires : les déchets des uns constituent des ressources pour les autres (Frosch et Gallopoulos, 1989 ; Frosch, 1992 ; Erkman, 2004).

4. Si ces auteurs appellent à intégrer les sciences humaines dans les recherches sur l'écologie industrielle, ils évoquent uniquement l'histoire, la sociologie, la psychologie et la géographie.

5. Les buts sont les "aspirations fondamentales sur un horizon temporel généralement non-borné précisément ». Par exemple : être leader sur le marché X ou améliorer la productivité des salariés (Desreumaux et al., 2009 : 11).

6. Les objectifs correspondent aux "résultats que l'entreprise se propose d'atteindre à une date donnée ». Par exemple: embaucher dix ingénieurs ou développer la formation des cadres au management (Desreumaux et al., 2009 : 11). Les objectifs sont mesurables (Ardoin et al., 2009).

7. Ardoin et al. (2009: 13) rappellent que « la distinction entre opportunités et menaces est souvent une pure question de point de vue."

8. "Si une organisation est légitime auprès des acteurs de son environnement, alors elle bénéficie d'un accès privilégié à des ressources » (Desreumaux et al., 2009 : 49).

9. En 2011, l'association Orée (Organisation pour le Respect de l'Environnement dans l'Entreprise) a recensé une trentaine de démarches éco-industrielles sur le territoire français.

10. De nombreux clubs d'entreprises proposent des collectes mutualisées de déchets à leurs adhérents. Les industriels, auparavant collectés individuellement, mettent en commun leurs déchets qui sont pris en charge par un prestataire unique. Ces pratiques permettent de rationnaliser et de réduire la consommation de ressources et les rejets polluants.

11. "Les motivations des individus peuvent être très différentes: protection de la santé et de celle des enfants, protection du paysage, protection de l'écosystème, protection du patrimoine immobilier [...]. Cet impact social peut se traduire par un rejet collectif du projet » explique Adoue (2007:5).

12. Pour un exemple, se référer au cas de la synergie entre Gyproc et Asnaesvaerket (symbiose de Kalundborg) présenté par Erkman (2004).

13. Au sein d'un écosystème naturel, il existe une redondance fonctionnelle quand coexistent plusieurs espèces capables d'assurer une même fonction. Cette redondance assure une certaine stabilité à l'écosystème et lui apporte une meilleure résistance aux perturbations (résilience) ainsi qu'une meilleure adaptabilité au changement.

14. Malgré le scepticisme de certains auteurs qui craignaient que la symbiose industrielle de Kalundborg reste figée, il semblerait que les partenaires aient réussi à évoluer (Chertow, 2007) : des innovations chez Statoil, Asnaesvaerket et Novo Nordisk ont été recensées.

15. L'organisation doit être gérée au seul profit des actionnaires.

16. Il s'agit de la traduction du terme "stakeholder » dérivé de "shareholder » selon Freeman (1999: 234).

17. "Une entité est légitime si ses actions sont perçues comme étant appropriées dans le cadre d'un système de normes ou de valeurs" (Desreumeaux et al., 2009 : 49).

18. Contrainte par les pouvoirs publics et les institutions (lois et réglementations), l'organisation adopte de nouvelles pratiques.

19. Face à l'incertitude, l'organisation copie des pratiques organisationnelles existantes qu'elle juge efficaces et légitimes.

20. L'organisation va suivre les normes découlant des communautés professionnelles et des réseaux associatifs auxquels elle appartient. 
21. Il s'agit de l'acceptabilité des pratiques éco-industrielles par les parties prenantes (syndrome "Not In My Back Yard"). L'entreprise doit veiller à ne pas se faire retirer son « droit d'exercer son activité ».

22. L'entreprise ne doit pas basculer dans l'opportunisme quant à la valorisation de ses matières résiduelles. Comment prend-elle en compte les risques sanitaires et environnementaux induits par les synergies éco-industrielles?

23. Comment les premières expériences françaises et internationales d'écologie industrielle ontelles conduit à la construction d'un cadre de référence en termes de méthodes et de pratiques ?

24. En quoi ce canevas peut-il contribuer à promouvoir l'adoption de telles pratiques mais aussi à enfermer les acteurs dans un modèle unique en limitant leur créativité ? Jusqu'où peut aller le mimétisme en matière d'écologie industrielle?

25. Ressources énergétiques disponibles, activité des entreprises en présence, dynamisme des pouvoirs publics, ...

26. En étudiant le processus de choix des agents, Simon (1961) a mis en évidence le rôle de l'information dans la prise de décision. Les agents ne disposent pas de toute l'information nécessaire (manque de temps, budget insuffisant) et sont contraints à prendre leur décision dans l'incertitude.

27. Poursuite de l'intérêt personnel par la ruse, la tromperie ou la rétention d'information (Ardoin et al., 2009).

28. "Des actifs sont dits spécifiques quand ils correspondent à un investissement durable devant être entrepris pour soutenir une transaction particulière, et que cet investissement n'est pas redéployable sur une autre transaction. » (Bouba-Olga, 2003 : 72).

29. Il s'agit de l'incertitude sur les conditions de réalisation de la transaction («incertitude environnementale »[Williamson, 1975] liée à l'environnement concurrentiel de la firme et « incertitude comportementale ou stratégique » [Williamson, 1985] liée à la difficulté d'anticiper le comportement d'autrui).

30. Caractère ponctuel ou récurent de la transaction.

31. Dyer et Singh (1998:662, notre traduction) définissent une rente relationnelle comme «un profit additionnel engendré conjointement par une relation d'échange qui ne peut être généré par aucune firme isolément. »

32. L'adhésion des entreprises à une association pratiquant l'écologie industrielle peut aussi être analysée. Dans quelle mesure ces associations de coordination (ex: Ecopal) réduisent-elles les coûts de transaction? Cette démarche représente également pour les entreprises une forme d'externalisation des tâches liées à l'environnement (recherche des prestataires, négociation des conditions de collecte et des tarifs pour les déchets,...). Quels sont les bénéfices de ce type d'arrangement (baisse des coûts opérationnels et d'organisation, recentrage sur le cœur de métier, accès à une expertise externe, mise en relation, ...) et les menaces qui en découlent (dépendance, perte du contrôle et d'information, ...).

33. En français : " modèle économique » ou « modèle d'affaires ».

34. Selon Soparnot et Grandval (2008), le développement durable peut permettre à l'entreprise de créer de la valeur (domination par les coûts ou différenciation).

35. Trois raisons peuvent expliquer la difficulté de duplication d'une ressource : des conditions historiques spécifiques à une firme (expérience unique développée sur une ressource); l'ambiguïté causale empêchant les concurrents de distinguer et imiter les ressources et les compétences valorisées par l'entreprise; et la complexité sociale (existence de savoir-faire tacites nécessaires à l'exploitation de la ressource).

36. Énergie présente ou piégée dans des matières ou des processus susceptible d'être valorisée. Par exemple, le potentiel énergétique des ordures ménagères est perdu lorsqu'elles sont incinérées sans récupération énergétique. 
37. Comment l'écologie industrielle intervient-elle au niveau de la chaîne de valeur interne (recherche et développement, approvisionnement, production) et de la chaîne de valeur externe (relations avec les partenaires éco-industriels) de l'organisation?

38. «Entrer dans un nouveau domaine d'activité » (Ardoin et al., 2009 : 434).

39. Dans quelle mesure, l'écologie industrielle peut-elle contribuer à développer certaines ressources (réputation, expérience, ...) ? Une telle démarche peut-elle mener à la construction et la valorisation de ressources et de compétences, permettant notamment le développement de nouvelles activités? Inversement, l'implémentation de l'écologie industrielle nécessite-t-elle des ressources et des compétences spécifiques?

40. Selon Weppe et al. (2012), les «ressources négatives" sont "perçues comme une faiblesse ou une source de coût par l'entreprise ». Comment l'entreprise peut-elle exploiter l'écologie industrielle pour trouver de nouveaux usages et créer de la valeur à partir de ressources «négatives » telles que les déchets?

\section{RÉSUMÉS}

Officiellement née dans les années 1990, l'écologie industrielle considère les déchets générés par les uns comme des matières premières pour les autres. Elle s'est progressivement imposée au sein de la communauté académique mais également auprès des entreprises et des pouvoirs publics. Si la nécessité de mobiliser les sciences humaines dans les recherches sur l'écologie industrielle est reconnue (Boons et Baas, 2006), le chercheur en stratégie est rarement représenté. Cette communication entend souligner les contributions potentielles du management stratégique au champ de l'écologie industrielle et esquisse un agenda de recherche.

For industrial ecology, officially born in the 1990s, waste produced by some processes or products can be reused as raw material by others. It gradually gained ground within the academic community, but also with companies and public authorities. If the need for human sciences to get involved in industrial ecology research has been acknowledged (Boons and Baas, 2007), the role of the researcher in strategy is often overlooked. This paper aims to highlight the potential contributions of strategic management to the field of industrial ecology and outlines a research agenda.

\section{INDEX}

Mots-clés : écologie industrielle, synergies éco-industrielles, coopération interentreprises, management stratégique, agenda de recherche, développement durable

Keywords : industrial ecology, eco-industrial synergies, inter-organizational cooperation, strategic management, research agenda, sustainable development 


\section{AUTEUR}

\section{ANNE-RYSLÉNE ZAOUAL}

Anne-Rysléne Zaoual est doctorante en Sciences de Gestion à l'IAE de Lille. Ses travaux portent principalement sur l'écologie industrielle et le développement durable du point de vue du management stratégique. Laboratoire LEM UMR CNRS 8179, anneryslenezaoual@yahoo.fr 